\title{
Evaluación de la reactividad árido-álcali en diversos áridos silicatados. Alternativas para minimizar esta reacción
}

\author{
Assessment of the alkali-silica reactivity in siliceous aggregates \\ and minimization alternatives
}

\author{
A. Martín¹, J. Gadea1, P.L. Campos¹, V. Calderón¹, M.A. García Calleja², A. Rodríguez¹
}

\section{RESUMEN}

Los áridos silíceos se caracterizan por reaccionar con el hidróxido cálcico liberado en el proceso de hidratación del cemento, lo que debido a su elevada avidez por el agua genera geles expansivos, que provocan importantes tensiones disruptivas en el seno del hormigón.

En este trabajo hemos evaluado la reactividad potencial de varios áridos silíceos (granitos, gneis, corneanas, cuarcita y serpentina) mediante el método acelerado de barras de mortero (normalizado), y el método de reactividad superficial, observando los geles silicocalcoalcalinos formados mediante microscopía electrónica de barrido (SEM).

Por otra parte, aportamos dos vías de minimización de esta reacción disruptiva, empleando ladrillo cerámico molido en un caso, y cemento CEM IV UNE-EN 197-1 en el otro.

Palabras clave: áridos silíceos, reacción árido-álcali, reactividad potencial, ladrillo cerámico molido.

\begin{abstract}
Siliceous aggregates are characterized for presenting certain chemical reactivity opposite to the calcium hydroxide liberated in the hydration of the Portland cement. The consequence of this reaction between the aggregate and the components of the intermediate concrete phase is the formation of gels very eager for water that can generate important disruptive pressures in the deeper structure of concrete. We have assessed the potential reactivity of several siliceous aggregates (granites, gneiss, hornfels, quartzite and serpentine) by means of the accelerated method in concrete bars (normalized method) and the superficial reactivity method, observing by scanning electron microscopy (SEM) the formation of silica calcium alkaline gels. On the other hand, we explore the way of minimizing this disruptive reaction employing ground clay bricks and cement type CEM IV UNE-EN 197-1.
\end{abstract}

Key words: siliceous aggregates, alkali-silica reaction, SEM, ground clay brick.

\section{Introducción}

La reacción álcali-silicato es un tipo de reacción árido-alcali que se produce cuando interaccionan áridos que contienen minerales silicatados potencialmente reactivos, con los álcalis del cemento (Stanton, 1948). Esta reactividad conlleva la formación de geles que muestran una gran avidez por el agua, lo que en presencia de humedad provoca su expansión (Mather, 1999; Menéndez \& Soriano, 2007), y como consecuencia de ésta agrietamientos $\mathrm{y}$, en definitiva, una disminución drástica de la durabilidad (Berube, 2002; Hobbs, 1988; Chrest et al., 1994).

En este trabajo se han empleado dos métodos para evaluar la reactividad potencial de áridos, el

\footnotetext{
1 Departamento de Construcciones Arquitectónicas e Ingeniería de la Construcción y del Terreno. Escuela Politécnica Superior. Universidad de Burgos, Burgos. Email: aitormd@ubu.es

2 Laboratorio Central de Estructuras y Materiales (CEDEX), Madrid.
} 


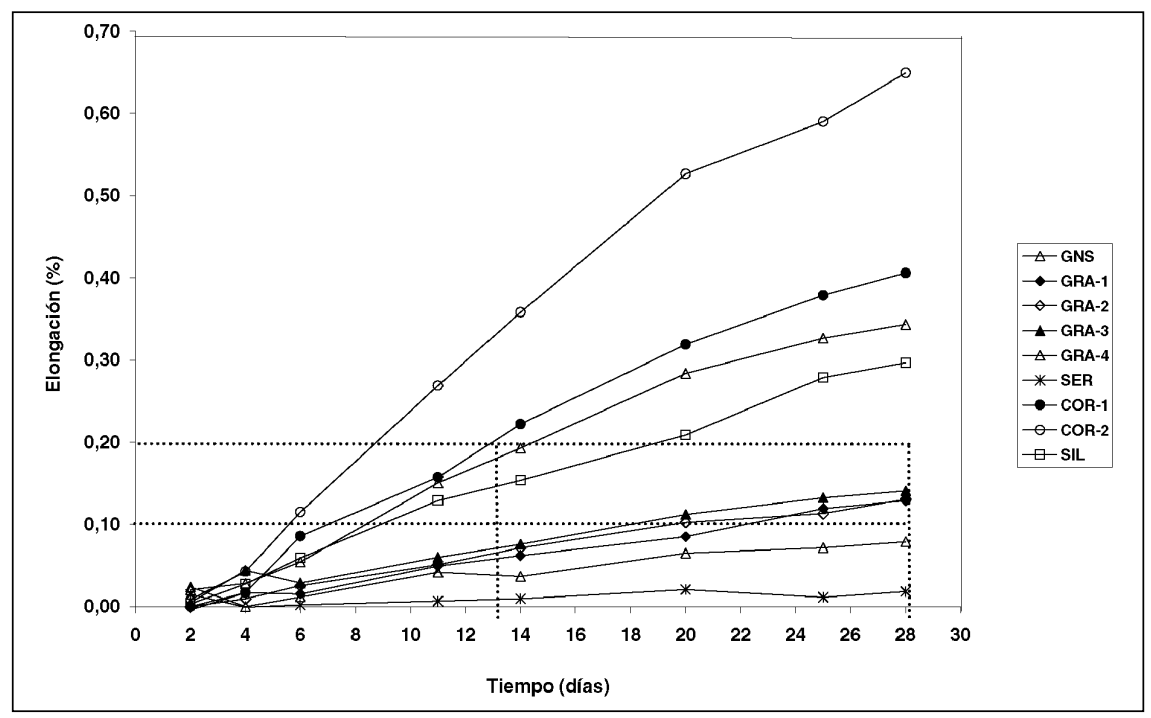

Fig. 1.-Elongaciones obtenidas en el ensayo acelerado de las barras de mortero.

primero normalizado y el segundo, que sin estarlo aún, ha sido, sin embargo, refrendado por diversas investigaciones centradas en este tipo de áridos:

- Ensayo acelerado en probetas de mortero. S/UNE-EN 146508:99 EX, empleando como arena los áridos estudiados y relacionando su reactividad con la expansión producida en dichas probetas tras ser atacadas con una disolución $1 \mathrm{~N}$ de $\mathrm{NaOH}$ a $80^{\circ} \mathrm{C}$.

- Método de reactividad superficial, utilizando difracción de rayos $\mathrm{X}$ y microscopía electrónica de barrido (SEM) para comprobar posibles cambios en la superficie del árido antes y después del ataque, y así poder comparar con los resultados del método normalizado.

Los áridos estudiados en este trabajo proceden de diversas canteras del suroeste de Castilla y León y de Galicia.

\section{Procedimientos experimentales y resultados}

\section{Estudio de los áridos. Descripción mineralógica}

La clasificación petrográfica de los áridos se ha realizado en base al anexo A de la norma UNE-EN 932-3: «Ensayos para determinar las propiedades generales de los áridos». Destaca la presencia en casi todos los áridos de feldespato potásico, cuarzo con extinción ondulante y varios tipos de micas (biotita y moscovita), minerales todos ellos de gran relevancia (por su inestabilidad) en la posterior reactividad álcali-silicato (U.S. Army, 2001).

\section{Método acelerado en probetas de mortero S/UNE-EN 146508:99 EX (ASTM C 1260-94, 1999)}

Procedimiento experimental: este análisis consiste en fabricar tres probetas de mortero de dimensiones $25 \mathrm{~mm}$ x $25 \mathrm{~mm}$ x $285 \mathrm{~mm}$, con cada uno de los áridos. El cemento utilizado es un CEM I 52,5R UNE 80301:1996, que contiene únicamente clínker y regulador de fraguado, sin ningún tipo de adiciones con carácter minoritario, de contenido en álcalis medio $\left(\mathrm{Na}_{2} \mathrm{O}_{\text {eq }} 0,74 \%\right)$. La relación cemento/árido es $1 / 2,25$, y la relación agua/cemento de referencia 0,47 , aunque en varios casos se aumenta para lograr la consistencia requerida en el ensayo (105-120 mm según UNE 83811). Las fracciones de árido empleadas se encuentran entre $5 \mathrm{~mm}$ y $0,16 \mathrm{~mm}$.

Estas probetas se someten al ataque de una disolución de $\mathrm{NaOH} 1 \mathrm{~N}\left(10^{3} \mathrm{~mol} \mathrm{~m}^{-3}\right)$ a $80{ }^{\circ} \mathrm{C}$ de temperatura, midiendo la elongación de cada probeta a diferentes edades. Se evalúa la dilatación lineal porcentual tanto a 14 como a 28 días, obteniendo mediante este método diferencias muy significativas de reactividad.

El resultado es que las dos corneanas, el gneis y la cuarcita resultan áridos reactivos (expansión a los 14 días mayor del $0,1 \%$, fig. 1). En el caso de las corneanas y el gneis, la presencia de feldespatos potásicos alterados a filosilicatos, y en la cuarcita el cuarzo con extinción ondulante, podrían ser la causa de la elevada reactividad, capaz de crear grietas en la superficie de las probetas (fig. 2). 


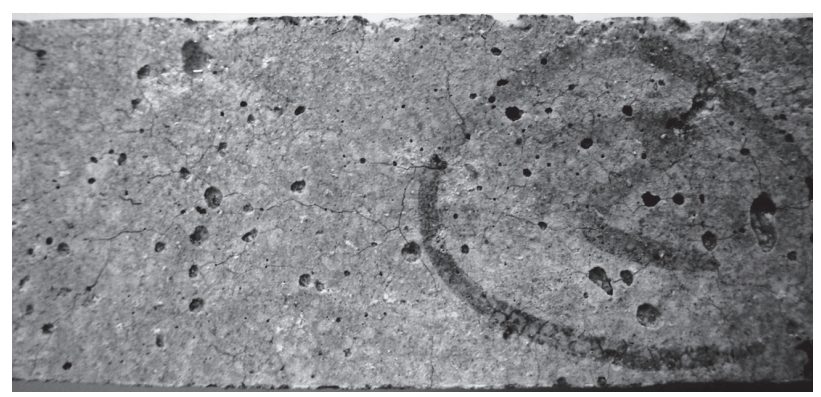

Fig. 2.-Fisuras superficiales en COR-1 provocadas por la expansión de los geles silicocalcoalcalinos.

Los datos obtenidos con este método resultan satisfactorios, aunque en el caso de áridos con expansiones próximas a la máxima establecida por la norma resulta conveniente prolongar el tiempo de ataque hasta los 28 días para asegurar resultados fiables. Debido en gran medida a la severidad del procedimiento, este método no admite dudas cuando clasifica a los áridos como no reactivos, pero si el resultado es el de árido reactivo es conveniente confirmar el resultado, que se lleva a cabo con el ensayo siguiente.

\section{Método de reactividad superficial: microscopía electrónica de barrido (SEM) y rayos $X$}

Procedimiento experimental: consiste en atacar en superficie fragmentos de áridos con una solución saturada de $\mathrm{Ca}(\mathrm{OH})_{2}$, es decir portlandita (compuesto alcalino liberado en el fraguado del cemento), estudiándose la misma superficie antes y después del ataque mediante microscopía electrónica de barrido acoplada a análisis por energías dispersivas de rayos X (SEM) (Leemann \& Holzer, 2005; Soriano, 1996).

Con este método se puede apreciar si los áridos son o no reactivos dependiendo de los efectos que la reacción árido-álcali ha causado en cada superficie en un punto determinado (Ben Haha et al., 2007; Gavrilenko et al., 2007). Por ejemplo, en el estudio de una de las corneanas se observa cómo se disuelve el cristal de biotita después del tratamiento alcalino (fig. 3). Por otro lado, comparando las superficies de los diferentes áridos se advierte que en los granitos se produce una importante sustitución de sílice por hidróxido cálcico. También la albita demuestra una elevada reactividad.

Por tanto, la observación de la textura superficial de los áridos antes y después del ataque denota la existencia de la interacción básica.

Se ha podido comprobar, tras observar fragmentos de las probetas de mortero con SEM, que el aumento de volumen obtenido en el método de las barras de mortero se debe a la aparición de geles (lisos o gruesos) calcoalcalinos producidos en el ataque básico.

Como ejemplo, pueden verse en la figura 4 dos de los compuestos, el primero con forma de «pétalos de rosa» y el segundo un gel grueso, ambos de naturaleza calcoalcalina, hallados en los áridos que han sufrido expansión, lo que demuestra que son potencialmente reactivos en ambientes básicos.

Por tanto, la observación al microscopio electrónico de las probetas de mortero y el método de reactividad superficial apoyan los resultados del método acelerado de probetas de mortero.

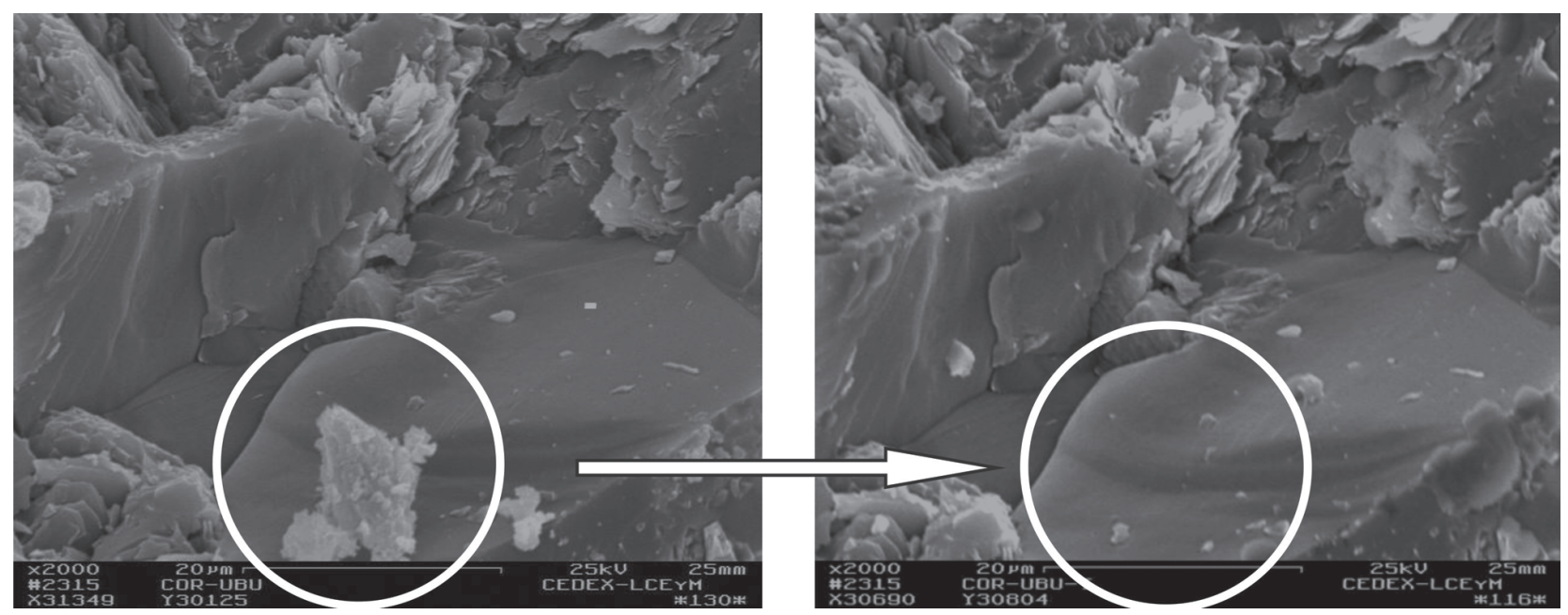

Fig. 3.-Disolución de cristal de biotita en COR-2 después del tratamiento básico. 

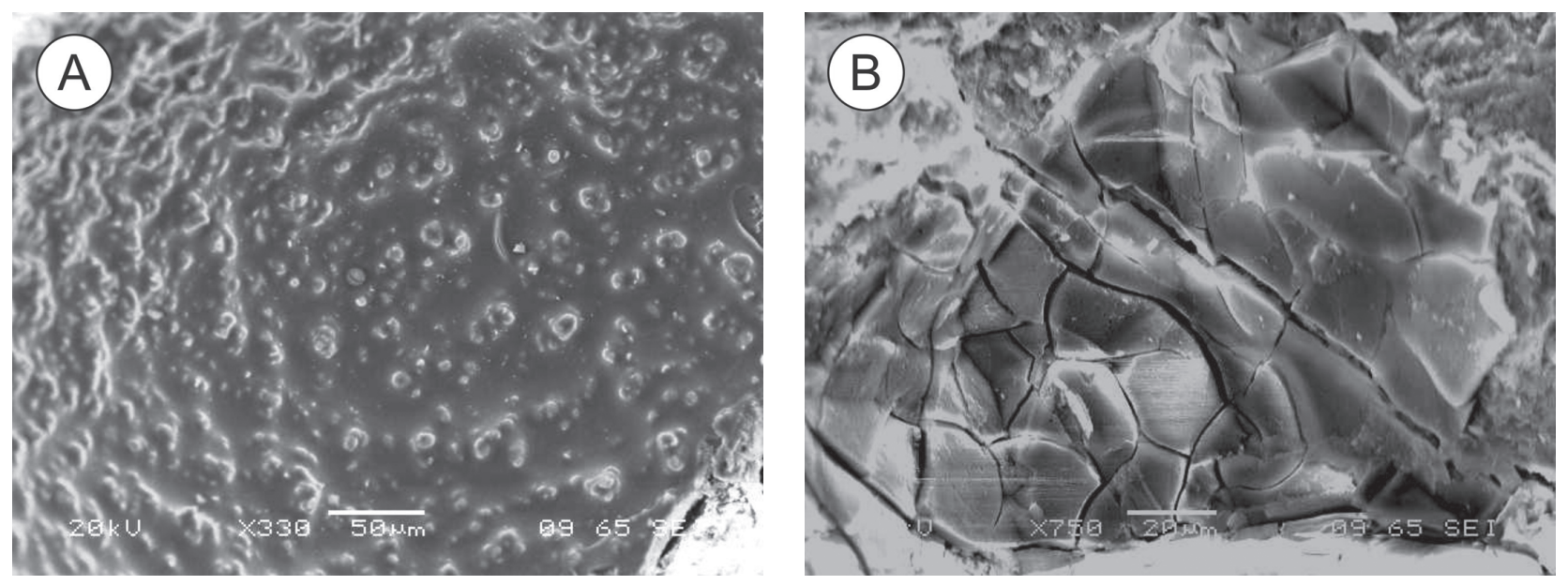

Fig. 4.-Geles silicocalcoalcalinos hallados en el análisis en superficie. A) Gel liso en COR-1. B) Gel grueso en COR-2.

\section{Reducción de la reactividad álcali-sílice}

Con el objeto de estudiar más profundamente el comportamiento de los áridos reactivos, se escogen tres de ellos para tratar de mitigar la formación de geles expansivos: una corneana, el gneis y la cuarcita.

Se pretende lograr la disminución de la reactividad aprovechando la conocida capacidad de los materiales puzolánicos de reaccionar con los álcalis generados en la hidratación del cemento, impidiendo de este modo la reacción con los áridos silicatados (Li \& Gress, 2006; Soriano, 1980; Thomas et al., 1997; Turanli et al., 2003).

En este trabajo se emplean dos medios sencillos de aportar puzolanicidad a los morteros:

- Añadiendo ladrillo cerámico molido de tamaño de grano inferior a $90 \mu \mathrm{m}$, aprovechando al mismo tiempo la condición de éstos como estériles acumulados en vertederos de todas las fábricas de materiales cerámicos. Se utiliza una dosificación de ladrillo molido del $25 \%$, que sustituye a un porcentaje similar de cemento.

- Fabricando los morteros con CEM IV (puzolánico) en vez de con CEM I, manteniendo idénticas las condiciones del ensayo acelerado en probetas de mortero.

Los resultados obtenidos avalan la hipótesis de que los materiales puzolánicos son capaces de reducir la expansión provocada por la reacción áridoálcali, aunque en diferente medida (fig. 5). La sustitución de CEM I con el $25 \%$ de ladrillo machacado logra mitigar en parte la expansión, no siendo, sin embargo, suficiente para superar el método acelerado en probetas de mortero. Por otra parte, los mor-

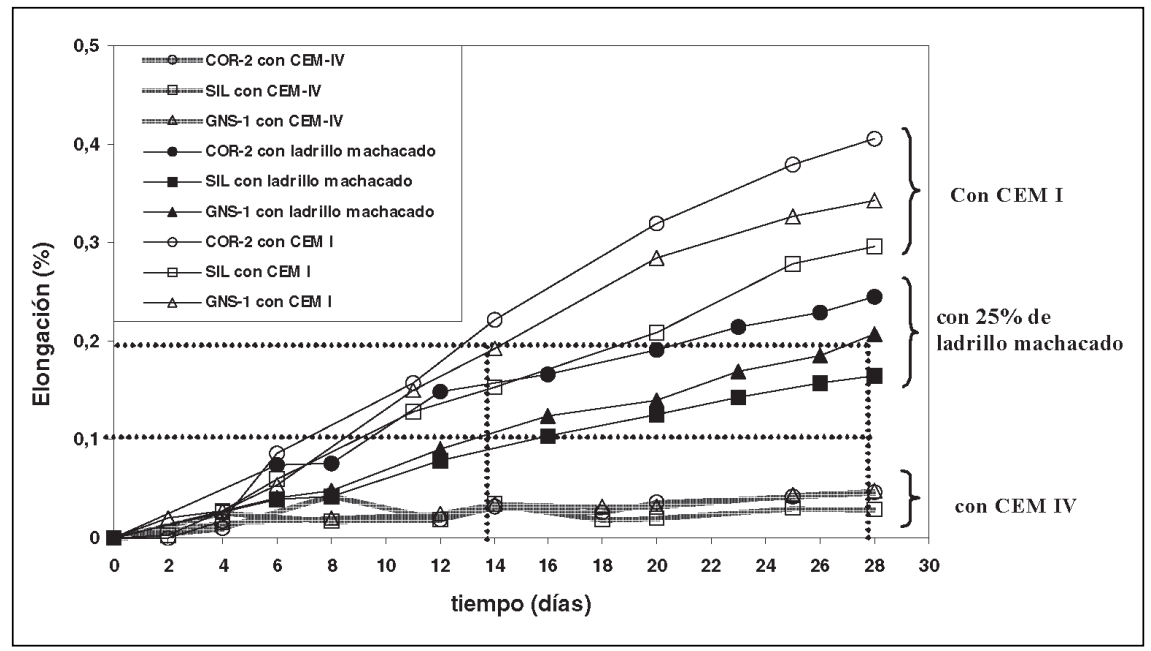

Fig. 5.-Reducción de las elongaciones empleando ladrillo machacado y CEM IV. 
teros fabricados con CEM IV logran una elevada reducción de la expansión, consiguiendo que los áridos anteriormente reactivos superen el ensayo acelerado incluso a los 14 días.

\section{Conclusiones}

El método acelerado de barras de mortero resulta ser un buen medio para evaluar la reactividad de los áridos alicatados, si es corroborado por el método superficial, que nos permite además describir la naturaleza química y morfológica de los geles expansivos formados.

El uso de materiales que presenten actividad puzolánica ha resultado ser una excelente vía para atenuar hasta niveles de elevada seguridad la expansión provocada por la reacción árido-álcali. Es imprescindible, sin embargo, estudiar además las consecuencias que sobre las propiedades mecánicas de los morteros conlleva el uso de estas alternativas puzolánicas, y estudiar la viabilidad de otras distintas.

El empleo de ladrillo machacado permitiría reutilizar este residuo de la fabricación de materiales cerámicos, aunque a la vista de los resultados de esta investigación es necesario ensayar otras proporciones y analizar sus consecuencias mecánicas.

\section{AGRADECIMIENTOS}

Los autores agradecen el apoyo económico prestado por la Junta de Castilla y León (Proyecto BU006B09), y por la Universidad de Burgos (Proyecto 2009/143); también agradecen la colaboración de la cementera Portland Valderrivas que ha suministrado los cementos utilizados en esta investigación.

\section{Referencias}

U.S. Army Corps of Engineers (2001). Engineering and design. Standard practices for concrete for civil works structures. U.S. Army Corps of Engineers, 122 págs.

ASTM C 1260-94 (1999). Standard test method for potential alkali reactivity of aggregates (mortar-bar method). Annual Book of ASTM Standards v. 04.02, Concrete and Aggregates, American Society for Testing and Materials, Philadelphia, 650-653.

Ben Haha, M.; Gallucci, E.; Guidoum, A. \& Scrivener, K.L. (2007). Relation of expansion due to alkali silica reaction to the degree of reaction measured by SEM image analysis. Cement and Concrete Research, 37: 1206-1214. doi:10.1016/j.cemconres.2007.04.016

Berube, M.A. (2002). Laboratory assessment of alkali contribution by aggregates to concrete and application to concrete structures affected by alkali-silica reacti- vity. Cement and Concrete Research, 32: 1215-1227. doi:10.1016/S00088846(02)007664

Gavrilenko, E.; García del Amo, D.; Calvo Pérez, B. \& García García, E. (2007). Comparison of ASR-gels in concretes against accelerated mortar bar test samples. Magazine of Concrete Research, 59: 483-494. doi:10.1680/macr.2007.59.7.483

Hobbs, D.W. (1988). Alkali-silica reaction in concrete. Ed. Thomas Telford, London, 192 págs.

Leemann, A. \& Holzer. L. (2005). Alkali-aggregate reaction. Identifying reactive silicates in complex aggregates by ESEM observation of dissolution features. Cement and Concrete Composites, 27: 796-801. doi:10.1016/j.cemconcomp.2005.03.007

Li, X.H. \& Gress. D.L. (2006). Mitigating alkali-silica reaction in concrete containing recycled concrete aggregate. Transportation Research Record, 1979: 30-35. doi:10.3141/1979-06

Mather, B. (1999). How to make concrete that will not suffer deleterious alkali-silica reaction. Cement and Concrete Research, 29: 1277-1280. doi:10.1016/S00088846(98)00240-3

Menéndez, E. \& Soriano, J. (2007). Procesos expansivos del hormigón: ensayos árido-álcali, ataque por sulfatos, hielo deshielo. Sistemas de prevención y actuaciones. Curso de Estudios Mayores de la Construcción, 17 edición. Instituto Eduardo Torroja, CSIC, Madrid.

Chrest, A.P.; Brown, R.; Breeze, P.C.; Clarke, J.L.F.; D'arcy, T.J.; Durning, T.; Eddy, D.B.; Gami, S.S.; Iverson, P.J.; Magnesio, C.; Meinheit, D.F.; Nadeau, F.A.; Peterson, C.A.; Rear, K.; Schupack, M. \& Walker, H.C. (1994). Alkali-aggregate reactivity: a summary. PCI Journal, 39: 26-35.

Soriano, J. (1980). Influencia de la naturaleza mineralógica de adiciones en el comportamiento de la pasta endurecida de cemento Portland. Revista de Obras Públicas, 127: 861-867.

Soriano, J. (1996). Aplicación del MEB al estudio de materiales de construcción. En: Microscopía electrónica de barrido y microanálisis por rayos X (Aballe, M.; López-Ruiz, J.; Badía, J.M. \& Adeva, P., eds.), Ed. Rueda, Madrid, 111-111.

Stanton, T.E. (1948). Correlation of laboratory tests with field experiences of excessive concrete expansion induced by a reaction between the cement and aggregate. Proceedings American Society for Testing and Materials, 48: 1057-1066.

Thomas, M.D.A.; Hooton, R.D. \& and Rogers. C.A. (1997). Prevention of damage due to alkali-aggregate reaction (AAR) in concrete construction. Canadian approach. Cement Concrete and Aggregates, 19: 26-30. doi:10.1520/CCA10018J

Turanli, L.; Bektas, F. \& Monteiro, P.J.M. (2003). Use of ground clay brick as a pozzolanic material to reduce the alkali-silica reaction. Cement and Concrete Research, 33: 1539-1542. doi:10.1016/S0008-8846(03)00101-7

Recibido el 26 de noviembre de 2009 Aceptado el 3 de febrero de 2010 Publicado online el 11 de mayo de 2010 